\section{Chronische Lyme-Borreliose: Gibt es die wirklich?}

In einer Spezialambulanz für die Lyme-Borreliose liegen bei $60 \%$ der dorthin überwiesenen Patienten keine Anhaltspunkte für eine bestehende Lyme-Krankheit vor, allerdings weisen die Patienten im Vergleich zur Allgemeinbevölkerung einen weit überproportionalen Anteil von Depressionen, Angststörungen und Persönlichkeitsstörungen auf.

Z wischen 2002 und 2007 unterzog man 240 Patienten, die sich auf Überweisung durch ihren Hausarzt oder auch durch eigene Initiative in einer Spezialambulanz für die Lyme-Borreliose vorstellten, neben einer ausführlichen anamnestischen Befragung, einer körperlichen Untersuchung und einschlägigen Laboruntersuchungen auch einer Reihe von strukturierten Tests zur Feststellung einer Depression, einer generalisierten Angststörung oder einer posttraumatischen Belastungsstörung. Die Bewältigung der Symptome wurde mit dem Coping-Strategies-Questionnaire und dem Fibromyalgia-Impact-Questionnaire evaluiert.

46 Patienten $(19,2 \%)$ hatten bei der Vorstellung eine aktive Infektion mit Borrelia burgdorferi und wurden entsprechend behandelt. Beim Follow-up berichteten sechs über anhaltende Symptome. Diese Patienten wurden zusammen mit 25 anderen, die bereits früher korrekt diagnostiziert und behandelt worden waren, dem Post-Lyme-Syndrom zugeordnet. 27 Patienten $(30 \%)$ stellten sich primär mit muskuloskeletären Beschwerden im Sinne eines Fibromyalgiesyndroms vor, die Symptome von 42 Patienten $(17,5 \%)$ mussten als medizinisch nicht erklärbar klassifiziert werden.

Die meisten Patienten wurden der Kategorie einer chronischen Multisystemerkrankung zugeordnet $(60,4 \%)$. Die

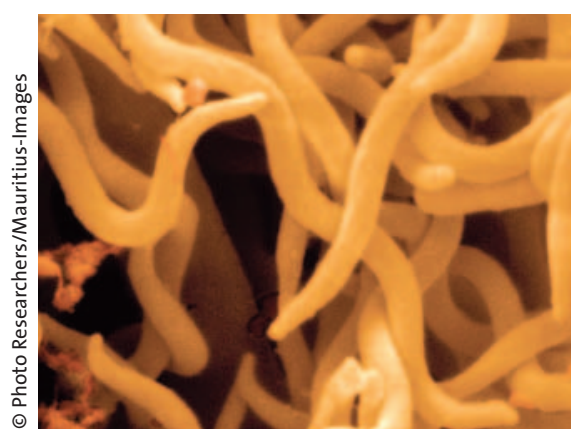

Symptome dieser Patienten konnten nicht durch eine Lyme-Borreliose erklärt werden. Sie wiesen aber im Vergleich zu den Patienten mit behandelter Lyme-Borreliose oder anderen medizinisch diagnostizierbaren Erkrankungen eine Odds Ratio von 3,54 für hohen negativen und geringen positiven Affekt sowie die Tendenz zum Katastrophisieren auf. Unter den psychiatrischen Diagnosen standen eine Major Depression und eine generalisierte Angststörung im Vordergrund.

Kommentar: Auch diese Untersuchung belegt, dass es das Krankheitsbild einer „chronischen Borreliose“ wahrscheinlich nicht gibt. Es handelt sich vielmehr um ein Hilfskonstrukt, um Patienten mit Angststörungen, Somatisierungsstörungen, Depressionen und Persönlichkeitsstörungen nicht mit dem ungeliebten Etikett einer psychiatrischen Erkrankung zu versehen. Auch einige Ärzte schwimmen auf dieser Welle mit großem Erfolg mit, sei es als Überzeugungstäter oder als Scharlatan.

H. S. FüeßI

Hassett AL et al. Psychiatric comorbidity and other psychological factors in patients with „Chronic Lyme Disease“. Am J Med 2009; 122: 843-50.

Borrelia burgdorferi: Wie erklären sich anhaltende Symptome?

\section{Bei Rosazea: Störung der Darmflora in Betracht ziehen}

Immer wieder wurden für die Rosazea Verbindungen mit Magen-DarmProblemen vermutet. Während es zu der Rolle von H. pylori widersprüchliche Daten gibt, wird mittlerweile eine bakterielle Fehlbesiedlung des Dünndarms (small intestinal bacterial overgrowth, SIBO) als möglicher pathogenetischer Faktor erwogen.

n dieser italienischen Studie stellte man bei 113 konsekutiven Patienten mit Rosazea im H2-Atemtest signifikant häufiger eine Überwucherung des Dünndarms mit Bakterien fest als bei nach Geschlecht und Alter sortierten Gesunden $(52$ der 113 Patienten gegenüber 3 der 60 Kontrollen). Die Patienten mit SIBO wurden entweder zehn Tage lang mit Rifaximin oder Placebo behandelt.
Die Intervention war nicht verblindet. In Fällen, in denen eine Eradikation gelang (bei 28 der 32 Verum-Patienten) heilten die Läsionen bei fast allen Patienten vollständig ab $(\mathrm{n}=20)$ bzw. besserte sich der Hautzustand erheblich $(n=6)$.

Nach einem Wechsel zu Rifaximin ließ sich die Fehlbesiedelung auch bei 17 der 20 Patienten aus dem Placebo-Arm korrigieren; 15 dieser Patienten wurden eben- falls völlig erscheinungsfrei. Die Remissionen blieben ohne jede andere Behandlung für mindestens neun Monate bestehen.

Ohne nennenswerten dermatologischen Nutzen war die antibiotische Behandlung dagegen in einer Gruppe von 16 Patienten mit negativem Atemtest.

Fazit: Die Ergebnisse sprechen für eine pathogenetische Bedeutung der Intestinalflora, speziell bei der Rosacea papulopustulosa. Die Autoren sehen in den Daten einen wichtigen Beitrag zum künftigen klinischen Management dieser oft schwierigen Fälle.

wpa

Parodi A et al. Small intestinal bacterial overgrowth in rosacea: clinical effectiveness of its eradication. Clin Gastroenterol Hepatol 2008; 6: 759-64. 